\title{
Alpha-fetoprotein testing for hepatocellular carcinoma may not be helpful in nonalcoholic steatohepatitis
}

\author{
Linda L. Wong ${ }^{1,2^{*}}$, Christopher J. Kim², Sandi A. Kwee ${ }^{3,4}$, Brenda Y. Hernandez ${ }^{3}$ \\ ${ }^{1}$ University of Hawaii Cancer Center, Honolulu, USA \\ ${ }^{3}$ Department of Surgery, University of Hawaii, John A. Burns School of Medicine, Honolulu, USA \\ ${ }^{2}$ Department of Medicine, University of Hawaii, John A. Burns School of Medicine, Honolulu, USA \\ ${ }^{4}$ PET Imaging Research, University of Hawaii, Honolulu, USA \\ Email: "hepatoma@aol.com
}

Received 21 October 2012; revised 22 November 2012; accepted 30 November 2012

\begin{abstract}
Background \& Objectives: Diagnosing hepatocellular carcinoma (HCC) often utilizes serum tumor markers. Although the most commonly used tumor marker in clinical practice, alpha-fetoprotein (AFP) is not included in recent guidelines for diagnosing HCC. The overall performance characteristics of AFP as a tumor marker is viewed as insufficiently sensitive or specific. The diagnostic value of AFP specifically in nonalcoholic steatohepatitis (NASH) related HCC is unknown. We aimed to determine the utility of AFP testing in NASH-related HCC. Methods: Retrospective review of 737 HCC patients referred from 19932011 to a single facility treating the majority of chronic liver disease in Hawaii. HCC was diagnosed histologically by percutaneous biopsy, liver biopsy at the time of surgery, or examination of the resected liver. Patients were classified according to $\mathrm{HCC}$ risk factors including NASH, hepatitis $B$ and $\mathrm{C}$ infection, and alcohol-related. Other data collected included: demographics, ethnicity, presence of cirrhosis, tumor characteristics (size, number, vascular invasion), diabetes, hyperlipidemia, body mass index (BMI) and blood testing to calculate Model for End-Stage Liver Disease (MELD) score. Elevated AFP was defined as $>20$ $\mathrm{ng} / \mathrm{mL}$. Sensitivity of AFP was determined and compared between various subgroups. Results: Elevated AFP levels were detected in $64.3 \%$ of patients. AFP sensitivity was $47 \%$ for NASH-related HCC $(n=100)$, and $67.2 \%$ for $\mathrm{HCC}$ with viral or alcoholic risk factors $(\mathrm{n}=637)(\mathrm{OR} 0.43,95 \%$ CI $0.28-0.66, \mathrm{p}=$ 0.0001). Elevated AFP had higher sensitivity in females $(71.9 \%$ vs. $61.8 \%$, OR $1.58,95 \%$ CI $1.1-2.27$, $p=0.013)$, non-diabetics $(67.4 \%$ vs. $57.2 \%$, OR 0.65 , $95 \%$ CI $0.47-0.89, p=0.0093)$, and cirrhotics $(67.1 \%$ vs. $56.8 \%$, OR $1.55,95 \%$ CI $1.10-2.19$, $p=0.0012$ ).
\end{abstract}

"Corresponding author.
AFP did not vary significantly with regard to hyperlipidemia or BMI. AFP was more sensitive in advanced disease including tumors $>5 \mathrm{~cm}$, multiple tumors, or vascular invasion (all with $\mathrm{p}<\mathbf{0 . 0 5}$ ). AFP did not vary with MELD score. Conclusions: Normal AFP is common in NASH-related HCC. Better tumor markers may be needed to optimally screen and diagnose NASH-related HCC. Without more effective tumor markers, HCC detection relies heavily upon imaging and liver biopsy.

Keywords: Hepatocellular Cancer; Nonalcoholic Steatohepatitis; Alpha-Fetoprotein

\section{INTRODUCTION}

Primary liver cancer is the fifth most common cancer worldwide, and the third leading cause of cancer death. $\mathrm{HCC}$ is the major histologic subtype among primary liver cancers. Endemic areas of HCC include Southeast Asia and sub-Saharan Africa [1]. In areas of low prevalence including North America and Europe, HCC incidence is rising $[2,3]$. In the United States, HCC had the highest increase in mortality, and second highest increase in incidence, of all cancers between 1995 and 2004 [4].

Chronic liver disease and cirrhosis are primary risk factors for HCC. Chronic viral infection with hepatitis B (HBV), hepatitis C (HCV), and alcoholic injury, are the predominant causes of liver disease [5]. In the United States, $\mathrm{HBV}$ rates are lower, whereas $\mathrm{HCV}$ is associated with $70 \%$ of HCC [6,7]. However, $15 \%-50 \%$ of HCC cases are non-viral and non-alcohol related and classified as cryptogenic [7-9].

Metabolic disease including obesity, diabetes mellitus, and hyperlipidemia, are associated with non-viral and non-alcohol related HCC [7]. Perhaps a hepatic manifestation of metabolic disease, non-alcoholic fatty liver disease (NAFLD) is the most common liver disease in the 
United States [10]. Characterizing a disease spectrum including simple steatosis, NASH, cirrhosis, and endstage liver disease (ESLD), NAFLD may progress to HCC, and account for a substantial portion of cryptogenic HCC [11,12].

Diagnosing HCC utilizes liver imaging, histopathologic evaluation, and tumor markers. Screening for HCC commonly uses liver ultrasound (US) and measuring AFP. Abnormal screening findings prompt diagnostic imaging with contrast-enhanced computed tomography (CT) and/or magnetic resonance imaging (MRI). Liver biopsy is used in scenarios with diagnostic ambiguity.

AFP is a glycoprotein which is normally produced by the fetal liver, yolk sac, and the gastrointestinal tract. Although it is most commonly elevated in HCC, elevations in serum AFP can be seen in various malignancies including testicular, bile duct, pancreatic, stomach, and colon cancer. Elevated AFP can also seen with non-malignant conditions including hepatitis and cirrhosis [13].

AFP is the most commonly used tumor marker for HCC in clinical practice. It is easily obtainable and relatively inexpensive. However, recent guidelines do not include AFP in the diagnostic algorithm because of its overall sensitivity or specificity for HCC [14]. Defining an elevated AFP level $>20 \mathrm{ng} / \mathrm{mL}$, confers a sensitivity of $60 \%$ and specificity of $80 \%$. At a cut-off AFP level > $200 \mathrm{ng} / \mathrm{mL}$, specificity approaches $100 \%$, but sensitivity falls to $20 \%$ [15]. Yet, these performance characteristics for AFP are based largely upon studies of patients with chronic viral hepatitis developing HCC. Adding to the uncertain diagnostic utility of AFP has been the observation that a significant number of small HCCs do not secrete AFP.

Although AFP has long been used as tumor marker for $\mathrm{HCC}$, it is increasingly viewed to be unnecessary in a contemporary role for diagnosis. Part of the reason for this change is likely due to improvements in diagnostic imaging. Another possibility may be the changing prevalence of chronic viral disease to fatty liver disease as the cause of chronic liver disease and HCC development. However, it is unclear whether there is a significant correlation between AFP and the development of HCC specifically in the setting of NASH. The purpose of this study is to compare the frequency of elevated AFP with various risk factors, and estimate the relative diagnostic utility of AFP in NASH-related HCC.

\section{METHODS}

This is a retrospective analysis of $737 \mathrm{HCC}$ cases referred to a Liver Center affiliated surgical group (LW) at Hawaii Medical Center-East (formerly St. Francis Medical Center) from 1993-2011. This medical center was a tertiary center and sole clinic dedicated to liver diseases in Hawaii, the only liver transplant center in the State, and the primary referral center for hepatobiliary surgery for American territories of the Pacific Basin (including American Samoa, Guam, Saipan, and the Marshall Islands). Additionally, a number of patients were foreign nationals from Asian countries, including China, Japan, Korea, and the Philippines, who sought medical care in the United States. This center managed approximately $60 \%-70 \%$ of HCC cases in the State of Hawaii.

HCC was diagnosed histologically by percutaneous biopsy, liver biopsy at the time of surgery, or examination of the resected liver. Consistent with the United Network for Organ Sharing (UNOS) policy regarding transplant for HCC, patients without histologic confirmation were diagnosed with $\mathrm{HCC}$ if they had chronic liver disease and a liver lesion $\geq 2 \mathrm{~cm}$ in size on two imaging studies (US, CT, or MRI) and one of the following: 1) vascular blush on CT or MRI 2) AFP $>200 \mathrm{ng} / \mathrm{ml}$, or 3 ) arteriogram confirming the tumor [16].

The clinical presentation (i.e. reasons for referral and clinical workup leading to the diagnosis of HCC) for each patient were categorized as 1) symptomatic (i.e. abdominal pain or mass, weight loss, liver decompensation, jaundice), 2) asymptomatic (e.g. workup prompted by incidental finding on a prior imaging test), and 3) asymptomatic - abnormal finding on screening.

Although the Liver Center at our institution recommended that patients with viral hepatitis and chronic liver disease undergo HCC screening with AFP testing and US every six months, there was no uniform practice within our community that lead to this cohort. However, patients referred based on screening results, were identified by AFP testing and/or imaging (either US, CT, or MRI) at various intervals, ranging from three to twelve months, consistent with National Comprehensive Cancer Network guidelines [17].

Demographic information, medical history, laboratory results, tumor characteristics, treatment, and survival data were collected via clinical interview by a single physician without structured questionnaire and entered into a prospective database. Information on diabetes mellitus, hyperlipidemia, smoking, family history of HCC, and risk factors for HCC including viral hepatitis, alcohol abuse (defined as greater than two alcoholic beverages daily for at least ten years), and other chronic liver diseases, were included. Patients who had elevated lipid levels or took a lipid-lowering agent were classified as having hyperlipidemia. Measured height and weight were used to determine body mass index (BMI), with obesity defined as BMI $\geq 30$. NASH was diagnosed based on liver biopsy, absence of significant alcohol use, negative hepatitis serologies, and no established etiology for liver disease after clinical work-up by a gastroenterologist and/or hepatologist.

Laboratory data collected included bilirubin level, al- 
bumin level, prothrombin time, creatinine, alanine aminotransferase (ALT), aspartate aminotranferase (AST), platelet count, and serum AFP level. Laboratory data used for the study were obtained on, or within 2 weeks, of the patient's initial visit. Bilirubin, prothrombin time with international normalized ratio (INR), and creatinine, were used to calculate the MELD score. Tumor size, number, and location, were used to determine the Tumor Node Metastases stage according to the American Joint Commission on Cancer (AJCC) staging manual [18]. Milan criteria was defined as having a single tumor $\leq 5 \mathrm{~cm}$ in size, or $\leq 3$ tumors each $\leq 3 \mathrm{~cm}$ in size, no macrovascular involvement, and no radiographic evidence of extrahepatic disease [19].

AFP levels were determined as normal $(\leq 20 \mathrm{ng} / \mathrm{mL})$ or elevated $(>20 \mathrm{ng} / \mathrm{mL})$. The proportion of elevated AFP was compared by ethnicity, race, hepatitis B and C status, and presence of NASH. Normal vs. elevated AFP were also analyzed by presence of cirrhosis, tumor size, vascular invasion, MELD score $\geq 10$ vs. MELD $<10$ and MELD $\geq 15$ vs. MELD $<15$.

Analyses were performed using Excel, and JMP 9.0 (SAS Institute). Group characteristics were compared using analysis of variance (continuous variables) and chisquared analysis (categorical variables) with calculation of odds-ratios and 95\% confidence intervals. Two-tailed p-values $<0.05$ were considered significant.

\section{RESULTS}

In this cohort of 737 patients, 551 patients $(74.8 \%)$ were male, and the mean age was 61.7 years. Ethnic distribution was: Asian-478 (64.9\%), White-136 (18.5\%) and Pacific Islander-103 (14.0\%). 20 patients were classi- fied "Other" and were Afro-American, Hispanic, or mixed ethnicity (see Table 1).

One hundred patients were diagnosed with NASH-related HCC. Their mean age was $67.2 \pm 11.0$ years, and the male: female ratio was 58:42. Compared to the 637 non-NASH patients, NASH-related HCC patients consisted of fewer males $(58.0 \%$ vs. $77.6 \%, \mathrm{p}<0.0001)$, more diabetics $(56.1 \%$ vs. $26.1 \%, \mathrm{p}<0.0001)$, large tumors $>5 \mathrm{~cm}$ in size $(62.0 \%$ vs. $46.8 \%, \mathrm{p}<0.01)$, and single tumors $(74.0 \%$ vs. $63.6 \%, \mathrm{p}<0.05)$. There was no difference in age, BMI, or MELD score between NASH and non-NASH patients. There was also no difference in the proportion of patients meeting the Milan Criteria.

The percentage of symptomatic patients at referral did not differ significantly between NASH and non-NASH patients (49\% vs. $40.5 \%, \mathrm{p}=0.127)$. However, patients with NASH-related HCC were less frequently identified through screening compared to non-NASH patients $(11.0 \%$ vs. $21.4 \%, p<0.05)$. Notably, a significantly lower percentage of NASH patients presented with elevated AFP levels compared to patients with diseases other than NASH $(47 \%$ vs. $67.2 \%, p=0.0001)$. While the mean AFP level did not differ significantly between NASH and non-NASH patients (12593 vs. $19721 \mathrm{ng} / \mathrm{mL}, \mathrm{p}=$ $0.15)$, the median AFP value in NASH was $14 \mathrm{ng} / \mathrm{mL}$ vs. $70 \mathrm{ng} / \mathrm{mL}$ in non-NASH patients.

With respect to metabolic disease, the frequency of elevated AFP did not differ in various groups categorized by BMI or the presence of hyperlipidemia. However, elevated AFP was less common in diabetics with HCC as compared to nondiabetics $(57.2 \%$ vs. $67.4 \%, \mathrm{p}=0.0093)$ (see Tables 2 and $\mathbf{3}$ ).

Elevated AFP were more frequent in patients with greater disease burden, including large tumors $(>5 \mathrm{~cm})$,

Table 1. AFP differences by gender and ethnicity.

\begin{tabular}{|c|c|c|c|c|}
\hline & Elevated AFP $>20 \mathrm{ng} / \mathrm{mL}(\mathrm{n}=474)$ & Normal AFP $\leq 20 \mathrm{ng} / \mathrm{mL}(\mathrm{n}=\mathbf{2 6 3})$ & Odds-ratio (95\% conf interval) & p-value \\
\hline Gender & & & & $\mathrm{p}=0.013$ \\
\hline Males & $341(61.8 \%)$ & $211(38.2 \%)$ & Ref & \\
\hline Females & $133(71.9 \%)$ & $52(28.1 \%)$ & $1.58(1.10-2.27)$ & \\
\hline \multicolumn{5}{|l|}{ Ethnicity ${ }^{*}$} \\
\hline White & $82(60.3 \%)$ & $54(39.7 \%)$ & Ref & \\
\hline Pacific Islander & $76(73.8 \%)$ & $27(26.2 \%)$ & $1.85(1.05-3.23)$ & \\
\hline Asian & $305(63.8 \%)$ & $173(36.2 \%)$ & $1.16(0.78-1.71)$ & \\
\hline Chinese & $59(61.5 \%)$ & $37(38.5 \%)$ & $1.05(0.61-1.79)$ & \\
\hline Filipino & $76(69.7 \%)$ & $33(30.3 \%)$ & $1.52(0.89-2.59)$ & \\
\hline Japanese & $115(59.6 \%)$ & $78(40.4 \%)$ & $0.97(0.62-1.52)$ & \\
\hline Korean & $30(66.7 \%)$ & $15(33.3 \%)$ & $1.32(0.64-2.68)$ & \\
\hline Southeast Asian & $20(71.4 \%)$ & $8(28.6 \%)$ & $1.65(0.68-4.00)$ & \\
\hline Age $<65$ years & $297(66.9 \%)$ & $147(33.1 \%)$ & $1.32(0.97-1.80)$ & $\mathrm{NS}(\mathrm{p}=0.08)$ \\
\hline Age $\geq 65$ years & $177(60.4 \%)$ & $116(39.6 \%)$ & Ref & \\
\hline Age $<50$ years & $78(71.6 \%)$ & $31(28.4 \%)$ & $1.47(0.94-2.30)$ & $\mathrm{NS}(\mathrm{p}=0.10)$ \\
\hline Age $\geq 50$ years & $396(63.1 \%)$ & $232(36.9 \%)$ & Ref & \\
\hline
\end{tabular}

"20 patients with "other ethnicity" not included in analysis. 
Table 2. AFP by risk factors.

\begin{tabular}{|c|c|c|c|c|}
\hline & Elevated AFP $>20 \mathrm{ng} / \mathrm{mL}$ & Normal AFP $\leq 20 \mathrm{ng} / \mathrm{mL}$ & Odds-ratio & p-value \\
\hline Hepatitis B & & & & $\mathrm{NS}(\mathrm{p}=0.94)$ \\
\hline Negative & $259(60.7 \%)$ & $168(39.3 \%)$ & Ref & \\
\hline Positive & $145(61.8 \%)$ & $92(38.8 \%)$ & $1.02(0.74-1.41)$ & \\
\hline Hepatitis C & & & & $\mathrm{p}<0.0001$ \\
\hline Negative & $278(61.1 \%)$ & $177(38.9 \%)$ & Ref & \\
\hline Positive & $231(83.7 \%)$ & $45(16.3 \%)$ & $3.27(2.25-4.74)$ & \\
\hline Alcohol & $207(65.7 \%)$ & $108(34.3 \%)$ & $1.11(0.82-1.51)$ & $\mathrm{NS}(\mathrm{p}=0.53)$ \\
\hline No Alcohol & $267(66.4 \%)$ & $155(33.6 \%)$ & Ref & \\
\hline NASH & $47(47 \%)$ & $53(53 \%)$ & $0.43(0.28-0.66)$ & $\mathrm{p}=0.0001$ \\
\hline Non-NASH & $428(67.2 \%)$ & $209(32.8 \%)$ & Ref & \\
\hline
\end{tabular}

Table 3. AFP by metabolic factors.

\begin{tabular}{|c|c|c|c|c|}
\hline & Elevated AFP $>20 \mathrm{ng} / \mathrm{mL}$ & Normal AFP $\leq 20 \mathrm{ng} / \mathrm{mL}$ & Odds-ratio & p-value \\
\hline Diabetic & $127(57.2 \%)$ & $95(42.8 \%)$ & $0.65(0.47-0.89)$ & $\mathrm{p}=0.0093$ \\
\hline Nondiabetic & $346(67.4 \%)$ & $167(32.6 \%)$ & Ref & \\
\hline Hyperlipidemia & $75(57.7 \%)$ & $55(42.3 \%)$ & $0.70(0.48-1.05)$ & $\mathrm{NS}(\mathrm{p}=0.09)$ \\
\hline No hyperlipidemia & $381(65.8 \%)$ & $198(34.2 \%)$ & Ref & \\
\hline $\mathrm{BMI}<20$ & $31(66 \%)$ & $16(34 \%)$ & Ref & $\mathrm{NS}(\mathrm{p}=0.066)$ \\
\hline $\mathrm{BMI} \geq 20$ & $331(61.1 \%)$ & $211(38.9 \%)$ & $0.81(0.43-1.52)$ & \\
\hline $\mathrm{BMI}<25$ & $147(65 \%)$ & $79(35 \%)$ & Ref & $\mathrm{NS}(\mathrm{p}=0.34)$ \\
\hline $\mathrm{BMI} \geq 25$ & $215(59.2 \%)$ & $148(40.8 \%)$ & $0.78(0.55-1.10)$ & \\
\hline $\mathrm{BMI}<30$ & $290(61.5 \%)$ & $179(38.5 \%)$ & Ref & $\mathrm{NS}(\mathrm{p}=0.75)$ \\
\hline $\mathrm{BMI} \geq 30$ & $72(60 \%)$ & $48(40.0 \%)$ & $0.93(0.61-1.39)$ & \\
\hline $\mathrm{BMI}<35$ & $339(61.5 \%)$ & $212(38.5 \%)$ & Ref & $\mathrm{NS}(\mathrm{p}=1.00)$ \\
\hline $\mathrm{BMI} \geq 35$ & $23(60.5 \%)$ & $15(39.5 \%)$ & $0.96(0.49-1.88)$ & \\
\hline
\end{tabular}

multiple tumors, and vascular invasion (see Table 4). The frequency of elevated AFP did not differ with regards to MELD score or cirrhotic disease.

Overall, 263 patients $(35.7 \%)$ had a normal AFP. The percentage of patients with an elevated AFP did not differ significantly with age as listed in Table 1, but was higher in females compared to males $(71.9 \%$ vs. $61.8 \%$, $\mathrm{p}=0.018$ ). An elevated AFP was also more common in Pacific Islanders $(73.8 \%)$ compared to Whites $(60.3 \%)$, although Asians and Asian subgroups did not differ compared to Whites (see Table 1 for odds-ratios). Elevated AFP did not differ with regard to HBV status, but was more frequently elevated in HCV-related HCC compared to HCV negative HCC (83.7\% vs. $61.1 \%)$. When patients were both $\mathrm{HBV}$ and $\mathrm{HCV}$ positive, $73.4 \%$ had elevated AFP. The frequency of elevated AFP did not differ depending on alcohol use.

\section{DISCUSSION}

Favorable outcomes in HCC are more likely when detected at an early stage. Thus, effective screening aims at detecting preclinical HCC. For this purpose, various combinations of AFP testing, imaging with US, CT and/or MRI, are recommended. The role of chronic viral hepatitis in hepatocarcinogenesis is established. Screening patients with chronic viral hepatitis has been shown to result in earlier HCC detection, and reduced cancer mortality [20]. Guidelines for screening and diagnosing HCC by the American Association for the Study of Liver Diseases (AASLD), and the European Association for the Study of the Liver (EASL), are largely based on studies involving viral-related HCC [14,21,22].

Non-viral related $\mathrm{HCC}$ is estimated to account for $15 \%-50 \%$ of all HCC cases [7]. NAFLD progressing to 
Table 4. AFP by tumor characteristics and underlying liver function.

\begin{tabular}{|c|c|c|c|c|}
\hline & Elevated AFP $>20 \mathrm{ng} / \mathrm{mL}$ & Normal AFP $\leq 20 \mathrm{ng} / \mathrm{mL}$ & Odds-ratio & p-value \\
\hline Single tumor & $286(60 \%)$ & $191(40 \%)$ & Ref & $\mathrm{p}=0.0012$ \\
\hline Multiple tumors & $184(72.2 \%)$ & $71(27.8 \%)$ & $1.73(1.24-2.41)$ & \\
\hline Largest tumor $<5 \mathrm{~cm}$ & $218(58 \%)$ & $158(42 \%)$ & Ref & $\mathrm{p}=0.0002$ \\
\hline Largest tumor $\geq 5 \mathrm{~cm}$ & $256(70.8 \%)$ & $104(29.2 \%)$ & $1.78(1.31-2.42)$ & \\
\hline No vascular invasion & $405(61.6 \%)$ & $253(38.4 \%)$ & Ref & $\mathrm{p}=0.001$ \\
\hline Vascular invasion & $69(87.3 \%)$ & $10(12.7 \%)$ & $4.31(2.18-8.52)$ & \\
\hline MELD $<15$ & $389(65.2 \%)$ & $207(34.8 \%)$ & Ref & $\mathrm{NS}(\mathrm{p}=0.39)$ \\
\hline MELD $\geq 15$ & $67(59.1 \%)$ & $43(40.9 \%)$ & $0.83(0.55-1.26)$ & \\
\hline MELD $<10$ & $264(64.9 \%)$ & $142(35.1 \%)$ & Ref & $\mathrm{NS}(\mathrm{p}=0.81)$ \\
\hline MELD $\geq 10$ & $192(64 \%)$ & $108(36 \%)$ & $0.96(0.70-1.31)$ & \\
\hline Non-cirrhotics & $105(56.8 \%)$ & $80(43.2 \%)$ & Ref & $\mathrm{p}=0.012$ \\
\hline Cirrhotics & $365(67.1 \%)$ & $179(32.9 \%)$ & $1.55(1.10-2.19)$ & \\
\hline
\end{tabular}

NASH-related HCC may contribute a significant portion of non-viral related HCC cases. NASH is estimated to effect $3 \%$ to $6 \%$ of the general population, and $30 \%$ of the morbidly obese [23]. However, it is clinically difficult to identify the population at risk with NASH, as many patients remain undiagnosed. Further, the degree to which NASH contributes to HCC risk is currently unknown. Those diagnosed with NASH may be followed by a primary care physician and not by a hepatologist or gastroenterologist expected to be more adherent to screening recommendations [24].

In the United States, a minority of patients undergo screening or surveillance before HCC is diagnosed. In one population based study, only $17 \%$ of HCC patients underwent surveillance before diagnosis [25]. In our study, screening was also infrequent, occurring in $11.0 \%$ of NASH patients, and $21.4 \%$ of non-NASH patients. Rather, our patients were commonly found to have HCC due to symptoms (NASH $49 \%$ and non-NASH patients $40.5 \%$ ), or by imaging performed for unrelated reason. Not surprisingly, data regarding HCC screening in NASH patients is lacking. There is less data regarding AFP testing in NASH-related HCC.

The primary finding of this study is the relative infrequency of an elevated AFP level in patients diagnosed with HCC with NASH as the only identified risk factor for their cancer. Indeed, the median AFP level of NASH patients diagnosed with HCC fell within the normal range in this study. This is somewhat surprising since the patients with NASH-related HCC were more likely in this study to have tumors $>5 \mathrm{~cm}$ in diameter, and were equally likely to have presented with symptoms, as compared to patients with HCC associated with other risk factors. In our patient population the majority of patients were not screened, and many presented with clinical symptoms, whether or not they had NASH. Thus, our data mainly reflects the efficacy of AFP as a confirmatory diagnostic test.

A primary limitation of this study was that it was a retrospective review of patients referred to a single center for HCC management. Although relative diagnostic sensitivities of elevated AFP levels for detecting HCC can be estimated based on the tables in this study, absence of a complete dataset that includes patients without HCC including those with NASH (i.e. those not referred for surgical consultation), limits formal estimations of diagnostic sensitivity, specificity, or overall accuracy. Furthermore, potential referral bias exists for any case series, although in this study, the geographic isolation of our patient population and our institution's status as the surgical referral center for the majority of HCC cases in our region, limit such potential sources of bias.

\section{CONCLUSION}

Our study cautions against reliance upon AFP as a screening tumor marker in patients suspected of having HCC, especially in those with NASH as the only risk factor. Without adequate tumor markers for $\mathrm{HCC}$ in NASH, other strategies are needed to enhance early detection. US and other imaging will likely remain the primary screening modalities for patients at risk. Our clinical practice continues to rely heavily upon increasing community awareness, imaging-based surveillance measures, and selective liver biopsy for early detection of HCC.

\section{REFERENCES}

[1] Ferlay, J., Shin, H.R., Bray, F., Forman, D., Mathers, C. and Parkin, D.M. (2010) Estimates of worldwide burden of cancer in 2008: GLOBOCAN 2008. International Journal of Cancer, 127, 2893-2917. doi:10.1002/ijc.25516 
[2] El-Serag, H.B. and Mason, A.C. (1999) Rising incidence of hepatocellular carcinoma in the United States. New England Journal of Medicine, 340, 745-750. doi:10.1056/NEJM199903113401001

[3] Gomaa, A.I., Khan, S.A., Toledano, M.B., Waked, I. and Taylor-Robinson, S.D. (2008) Hepatocellular carcinoma: Epidemiology, risk factors and pathogenesis. World Journal of Gastroenterology, 14, 4300-4308. doi:10.3748/wig. 14.4300

[4] US National Institute of Health (2010) SEER Cancer Statistics Review 1975-2004.

http://www.SEER.cancer.gov

[5] Perz, J.F., Armstrong, G.L., Farrington, L.A., Hutin, Y.J. and Bell, B.P. (2006) The contributions of hepatitis B virus and hepatitis $\mathrm{C}$ virus infections to cirrhosis and primary liver cancer worldwide. Journal of Hepatology, 45, 529-538. doi:10.1016/j.jhep.2006.05.013

[6] Hassan, M.M., Frome, A., Patt, Y.Z. and El-Serag H.B. (2002) Rising prevalence of hepatitis $C$ virus infection among patients recently diagnosed with hepatocellular carcinoma in the United States. Journal of Clinical Gastroenterology, 35, 266-269.

doi:10.1097/00004836-200209000-00013

[7] El-Serag, H.B. (2004) Hepatocellular carcinoma: Recent trends in the United States. Gastroenterology, 127, S27S34. doi:10.1053/j.gastro.2004.09.013

[8] Marrero, J.A., Fontana, R.J., Su, G.L., Conjeevaram, H.S., Emick, D.M. and Lok A.S. (2002) NAFLD may be a common underlying liver disease in patients with hepatocellular carcinoma in the United States. Hepatology, 36, 1349-1354. doi:10.1002/hep.1840360609

[9] Starley, B.Q., Calcagno, C.J. and Harrison, S.A. (2010) Nonalcoholic fatty liver disease and hepatocellular carcinoma: A weighty connection. Hepatology, 51, 1820-1832. doi:10.1002/hep.23594

[10] Ong, J.P. and Younossi, Z.M. (2007) Epidemiology and natural history of NAFLD and NASH. Clinics in Liver Disease, 11, 1-16. doi:10.1016/j.cld.2007.02.009

[11] Page, J.M. and Harrison, S.A. (2009) NASH and HCC. Clinics in Liver Disease, 13, 631-634. doi:10.1016/j.cld.2009.07.007

[12] Baffy, G., Brunt, E.M. and Caldwell, S.H. (2012) Hepatocellular carcinoma in non-alcoholic fatty liver disease: An emerging menace. Journal of Hepatology, 56, 13841391. doi:10.1016/j.jhep.2011.10.027

[13] Di Bisceglie, A.M., Sterling, R.K., Chung, R.T., Everhart, J.E., Dienstag, J.L., Bonkovsky, H.L., Wright, E.C., Everson, G.T., Lindsay, K.L., Lok, A.S., Lee, W.M., Morgan, T.R., Ghany, M.G. and Gretch, D.R. (2005) Serum alphafetoprotein levels in patients with advanced hepatitis $\mathrm{C}$ : Results from the HALT-C Trial. Journal of Hepatology,

\section{3, 434-441. doi:10.1016/j.jhep.2005.03.019}

[14] Bruix, J. and Sherman, M. (2005) Management of hepatocellular carcinoma: An update. Hepatology, 42, 12081236. doi: $10.1002 /$ hep. 20933

[15] Gonzalez, S.A. and Keeffe, E.B. (2011) Diagnosis of heaptocellular carcinoma: role of tumor markers and liver biopsy. Clinics in Liver Disease, 15, 297-306. doi:10.1016/j.cld.2011.03.012

[16] United Network for Organ Sharing (2011) Policy 3.6.4.4. www.unos.org

[17] National Comprehensive Cancer Network (2010) NCCN Clinical Practice Guidelines in Oncology: Hepatobiliary Cancers. V.2.2010.

[18] Edge, S.B. (2010) American Joint Committee on Cancer staging manual. 7th Edition, Springer, New York.

[19] Mazzaferro, V., Regalia, E., Doci, R., Andreola, S., Pulvirenti, A., Bozzetti, F., Montalto, F., Ammaturna, M., Morabito, A. and Gennan, L. (1996) Liver transplantation for the treatment of small hepatocellular carcinomas in patients with cirrhosis. New England Journal of Medicine, 334, 693-699. doi:10.1056/NEJM199603143341104

[20] Zhang, B.H., Yang, B.H. and Tang, Z.Y. (2004) Randomized controlled trial of screening for hepatocellular carcinoma. Journal of Cancer Research in Clinical Oncology, 130, 417-422. doi:10.1007/s00432-004-0552-0

[21] Bruix, J., Sherman, M., Llovet, J.M., Beaugrand, M., Lencioni, R., Burroughs, A.K., Christensen, E., Pagliaro, L., Colombo, M. and Rodés J. (2001) Clinical management of hepatocellular carcinoma. Conclusions of the Barcelona 2000 EASL conference. European Association for the Study of the Liver. Journal of Hepatology, 35, 421-430. doi:10.1016/S0168-8278(01)00130-1

[22] Bruix, J. and Sherman, M. (2011) Management of hepatocellular carcinoma: An update. Hepatology, 53, 10201022. doi:10.1002/hep. 24199

[23] Torres, D.M. and Harrison, S.A. (2008) Diagnosis and therapy of nonalcoholic steatohepatitis. Gastroenterology, 134, 1682-1698. doi:10.1053/j.gastro.2008.02.077

[24] Patwardhan, V., Paul, S., Corey, K.E., Mazhar, S.M., Richter, J.M., Thiim, M. and Chung R.T. (2011) Hepatocellular carcinoma screening rates vary by etiology of cirrhosis and involvement of gastrointestinal sub-specialists. Digestive Disease Sciences, 56, 3316-3322. doi:10.1007/s10620-011-1836-2

[25] Davila, J.A., Morgan, R.O., Richardson, P.A., Du, X.L., McGlynn, K.A. and El-Serag, H.B. (2010) Use of surveillance for hepatocellular carcinoma among patients with cirrhosis in the United States. Hepatology, 52, 132141. doi:10.1002/hep. 23615 